\title{
The Structure and Composition of Cartilage Keratan Sulphate
}

\author{
By JOHN J. HOPWOOD* and H. CLEM ROBINSON \\ Department of Biochemistry, Monash University, Clayton, Vic. 3168, Australia
}

(Received 18 February 1974)

\begin{abstract}
Keratan sulphate was isolated from bovine intervertebral disc and bovine nasal septum after hydrolysis with proteinases and treatment with dilute alkali. Each preparation was found to contain, per keratan sulphate chain: (a) 1 residue of mannose; (b) 3 residues of $\mathrm{N}$-acetylneuraminic acid (2 residues after alkali treatment); (c) 1 residue of $N$ acetylgalactosamine (lost after alkali treatment); $(d) 1$ residue or less of fucose. $N$-Acetylneuraminic acid residues were at non-reducing termini and were bonded to keratan sulphate through galactose residues. Evidence is presented for two different types of linkage between skeletal keratan sulphate and protein. Consideration of molecular parameters and compositions leads to a proposed structure for keratan sulphate-protein as found in skeletal proteoglycans.
\end{abstract}

The repeating disaccharide structure in keratan sulphate is composed of alternating galactose and $N$-acetylglucosamine residues linked $\beta-1,4$ and $\beta-1,3$ respectively. This repeating sequence is sulphated to various degrees at the C-6 position of both sugar residues (Bhavanandan \& Meyer, $1966,1967,1968)$. The repeating sequence has been used to classify the polysaccharide keratan sulphate into the same category as other connective-tissue glycosaminoglycans. However, keratan sulphate, compared with these glycosaminoglycans, has a considerably more complex structure. In most keratan sulphate preparations there is a molar excess of galactose over glucosamine. Bhavanandan \& Meyer (1968) have suggested that these extra galactose residues are linked as side branches from the keratan sulphate chain, although their exact location has yet to be established. Other sugars that appear to be component parts of the keratan sulphate structure are fucose, mannose, sialic acid and $\mathrm{N}$-acetylgalactosamine (Gregory \& Rodén, 1961; Mathews \& Cifonelli, 1965; Seno et al., 1965; Bray et al., 1967). The exact location of fucose and sialic acid residues within the keratan sulphate structure is unknown. They appear, however, to occupy terminal positions on side branches off the main keratan sulphate chain (Bhavanandan \& Meyer, 1967, 1968; Luscombe $\&$ Phelps, 1967b). There is pronounced variation in the content of fucose and sialic acid in keratan sulphate isolated from tissues of different age and origin, or within fractions prepared from a single tissue (Seno et al., 1965; Mathews \& Cifonelli, 1965; Toda \& Seno, 1970; Nakagawa, 1971). It is not known if this variation reflects a natural heterogeneity of keratan sulphate composition introduced during

* Present address: Department of Pediatrics, University of Chicago, Chicago, Ill. 60637, U.S.A. its biosynthesis, or stems from hydrolysis of labile sugars during isolation and purification. Bray et al. (1967) have shown that cartilage keratan sulphate is linked to protein via an $\mathrm{O}$-glycosidic $\mathrm{N}$-acetylgalactosaminyl-serine or -threonine bond and that the remainder of the keratan sulphate chain is linked to the C-6 position of this $N$-acetylgalactosamine residue. Hopwood \& Robinson (1974) reported that a disaccharide, $\mathrm{N}$-acetylneuraminylgalactose, is linked to position 3 of this linkage-region $\mathrm{N}$-acetylgalactosamine. Baker et al. (1959) have suggested that mannose is located close to this linkage region to protein. Besides the $O$-glycosidic linkage with protein, cartilage keratan sulphate also appears to be bound to protein through an alkali-stable bond (Seno et al., 1965; Meyer, 1969, 1970; Pedrini, 1969; Heinegård, 1972).

In the present paper the composition of several cartilage keratan sulphate preparations is presented and a detailed structure for keratan sulphate is proposed.

\section{Experimental}

Preparation of keratan sulphate products KS-P and $K S-A$

Keratan sulphate preparations were isolated from adult bovine intervertebral disc after digestion with Pronase, papain and chondroitin sulphate lyase as described previously (preparation KS-2; Hopwood \& Robinson, 1974). Such preparations are designated KS-P to indicate isolation by proteolysis.

Keratan sulphate was also isolated from milled cartilage samples as outlined by Hopwood \& Robinson (1973). Cartilage (3g dry wt.) was stirred with $50 \mathrm{ml}$ of $0.02 \mathrm{M}-\mathrm{NaB}^{3} \mathrm{H}_{4}$ in $0.5 \mathrm{M}-\mathrm{KOH}$ at $4^{\circ} \mathrm{C}$ for 10 days. The reaction mixture was 
acidified with acetic acid, desalted on Bio-Gel P-4 and fractionated by ion-exchange chromatography on ECTEOLA-cellulose (Robinson \& Hopwood, 1973). All of the keratan sulphate, together with chondroitin sulphate, was located in the most retarded peak of radioactivity. Chondroitin sulphate was removed from this fraction by digestion with chondroitin sulphate lyase followed by chromatography on Sephadex G-50 (Robinson \& Hopwood, 1973). The keratan sulphate fraction was then reapplied to the ECTEOLA-cellulose column and eluted with a linear $\mathrm{NaCl}$ gradient generated from $500 \mathrm{ml}$ of imidazole- $\mathrm{HCl}$ buffer $\left(0.02 \mathrm{M}\right.$ in $\left.\mathrm{Cl}^{-}\right)$ pH 5.7 and $500 \mathrm{ml}$ of $2.0 \mathrm{M}-\mathrm{NaCl}$ in $0.02 \mathrm{M}$-imidazole$\mathrm{HCl}$ buffer, $\mathrm{pH}$ 5.7. The hexose-positive and radioactive material was eluted as a single peak and desalted on a column of Bio-Gel P-4 and freeze-dried. Preparations isolated by this method are designated as KS-A to indicate alkali treatment.

\section{Acetic acid hydrolysis of the keratan sulphate product $K S-A$}

A sample of preparation KS-A $(50 \mathrm{mg})$ from adult bovine intervertebral disc was dissolved in $5 \mathrm{ml}$ of $1 \mathrm{M}$-acetic acid, sealed in a glass tube under vacuum and heated at $108^{\circ} \mathrm{C}$ for $4 \mathrm{~h}$. The hydrolysate was neutralized with pyridine and applied to a column $(95 \mathrm{~cm} \times 2.7 \mathrm{~cm}$ diam.) of Sephadex G-25 equilibrated with pyridine-acetic acid buffer, $\mathrm{pH} 5.2(0.2 \mathrm{M}$ in acetic acid). The column was eluted with the same buffer at $60 \mathrm{ml} / \mathrm{h}$ and $12 \mathrm{ml}$ fractions were collected and assayed for protein and hexose content and radioactivity. Fractions 15-20 (Fig. 1) were combined, evaporated under vacuum at room temperature to approx. $4 \mathrm{ml}$, diluted with $6 \mathrm{ml}$ of Tris- $\mathrm{HCl}$ buffer, pH 7.9 $\left(0.1 \mathrm{M}^{\text {in }} \mathrm{Cl}^{-}\right)$and again digested with Pronase. The digest was applied to and eluted from the same column $(80 \mathrm{~cm} \times 2 \mathrm{~cm})$ of Sephadex G-50 and eluted with $0.2 \mathrm{M}$-pyridine acetate buffer, $\mathrm{pH} 5.2$, at $48 \mathrm{ml} / \mathrm{h}$ (fractions were $12 \mathrm{ml}$ ). All of the applied hexose-positive material was recovered in fractions 12-17, which when combined and freeze-dried yielded $12 \mathrm{mg}$ of dry product. This material is designated preparation KS-A-A.

\section{Alkaline treatment of product $K S-P$}

A solution of $50 \mathrm{mg}$ of product $\mathrm{KS}-\mathrm{P}$ in $50 \mathrm{ml}$ of $0.02 \mathrm{M}-\mathrm{NaB}^{3} \mathrm{H}_{4}$ in $0.5 \mathrm{M}-\mathrm{KOH}$ was flushed with $\mathrm{N}_{2}$ and stirred under $\mathrm{N}_{2}$ at $4^{\circ} \mathrm{C}$ for 10 days. The reaction mixture was acidified with acetic acid, freeze-dried and dissolved in $1 \mathrm{ml}$ of $0.2 \mathrm{M}$-pyridine acetate buffer, pH5.2. This solution was then fractionated on a column of Sephadex G-25 and the void-volume fractions were further separated on a column of Sephadex G-50 into keratan sulphate and chon- droitin sulphate linkage-region fractions (Hopwood \& Robinson, 1974). The keratan sulphate preparation isolated by this method is designated KS-P-A.

\section{Enzymic digestion of keratan sulphate}

Galactose oxidase from Polyporus circinatus (Miles Laboratories, Kankakee, Ill., U.S.A.) together with horseradish peroxidase (Calbiochem, Los Angeles, Calif., U.S.A.) was used as described by Sempere et al. (1965). $\beta$-Galactosidase from Escherichia coli (C. F. Boehringer und Soehne G.m.b.H., Mannheim, Germany) or from Helix pomatia (Industrie Biologique Française S.A., Paris, France) was coupled with galactose dehydrogenase (C. F. Boehringer und Soehne) as described by Lindahl \& Rodén (1965). Leucine aminopeptidase from hog kidney (Sigma Chemical Co., St. Louis, Mo., U.S.A.) and carboxypeptidase A (Sigma Chemical Co.) were used as described by Smith (1955) and Neurath (1955) respectively.

Neuraminidase from Vibrio cholerae (50EC units; Farbwerke-Hoechst B.A.A., Frankfurt-Main, Germany) was incubated for $6 \mathrm{~h}$ at $37^{\circ} \mathrm{C}$ with keratan sulphate (approx. $400 \mu \mathrm{g}$ of sialic acid) in $10 \mathrm{ml}$ of $0.05 \mathrm{M}$-sodium acetate, pH5.5, containing $\mathrm{NaCl}$ $(9 \mathrm{mg} / \mathrm{ml})$ and $\mathrm{CaCl}_{2}(1 \mathrm{mg} / \mathrm{ml})$. This digest was fractionated on a column of Sephadex G-50 into free sialic acid and keratan sulphate.

Analytical methods have been described (Robinson \& Hopwood, 1973; Hopwood \& Robinson, 1974).

\section{Results}

The compositions and molecular weights of a number of preparations of keratan sulphate are shown in Table 1. A preparation designated KS-P, derived from adult bovine intervertebral disc by sequential digestion with Pronase, papain and chondroitin sulphate lyase, has been shown to consist of single chains of keratan sulphate linked via a peptide to a small chondroitin sulphate linkage-region oligosaccharide (Hopwood \& Robinson, 1974). Preparations designated KS-A, isolated by alkali extraction of cartilage, contain, on the other hand, single chains of keratan sulphate free of chondroitin sulphate fragments (Table 1). The average molecular weight $\left(\bar{M}_{n}\right)$ of each preparation was estimated by using end-group assay, which assumes that reduction of keratan sulphate with $\mathrm{NaB}^{3} \mathrm{H}_{4}$ introduces one ${ }^{3} \mathrm{H}$ atom/ polysaccharide chain. Similar molecular-weight values for the keratan sulphate preparations KS-P and KS-A from adult intervertebral disc are obtained by the end-group-assay method and by equilibrium sedimentation (Table 1). This result is consistent 


\section{Table 1. Analytical results and molecular-weight values for keratan sulphate preparations}

Results are expressed as percentage by weight of the dry sodium salt of each preparation. Galactosamine content is expressed as a molar ratio to glucosamine. KS-A preparations were isolated by alkaline cleavage and KS-P preparations by exhaustive digestion with papain, Pronase and chondroitin sulphate lyase as outlined in the Experimental section. Adult tissues were obtained from steers (2-5 years) within $1 \mathrm{~h}$ of slaughter and foetal tissues were obtained from embryonic calves obtained from the slaughterhouse just before full term. The assay methods for hexosamine, hexose, sulphate, hexuronate and sialic acid (as $N$-acetylneuraminic acid) have been described (Hopwood \& Robinson, 1974). The molar ratio galactosamine/ glucosamine was determined with a Beckman Spinco automatic amino acid analyser (Hopwood \& Robinson, 1974). Protein was determined by the method of Lowry et al. (1951) with dried crystalline bovine serum albumin (Commonwealth Serum Laboratories, Melbourne, Australia) as standard; the values in parentheses were calculated from results of amino acid analysis. Molecular weights were determined (a) by end-group estimation with $\mathrm{NaB}^{3} \mathrm{H}_{4}$ (Hopwood \& Robinson, 1973, 1974), (b) by analytical fractionation on Sephadex G-200 (Hopwood \& Robinson, 1973) and (c) by equilibrium sedimentation (Robinson \& Hopwood, 1973). ND, Not determined; nil, not detected.

Sample no. ... 1

Tissue source ... Adult bovine

Adult bovine KS-A

Type of preparation Composition $(\%)$

Hexosamine

Hexose

Sulphate

Protein

Hexuronate

Glucosamine/galactosamine

Sialic acid

$10^{-3} \times$ mol.wt.

$$
\bar{M}_{\mathrm{n}}(a)
$$

$\vec{M}_{\mathrm{n}}(b)$

$\bar{M}_{\mathrm{w}}(b)$

$\bar{M}_{\mathrm{z}}($ b)

$\bar{M}_{\mathrm{w}}(c)$

\section{6}

28.7

17.1

5.3

(7.2)

nil

100

3.3

20.5

21.9

24.8

28.5

18.6
3

Foetal bovine Adult bovine rtebral disc intervertebral disc nasal septum KS-P KS-A KS-A

28.0

32.2

18.0

4.6

(7.4)

3.2

4.4

4.7

17.9

ND

ND

ND

18.2
22.5

27.4

11.0

ND

nil

ND

7.3

10.0

10.6

12.2

14.8

ND
5

Foetal bovine nasal septum

KS-A

22.2

30.0

9.1

ND

ND

ND

11.0

4.0

ND

ND

ND

ND with the assumptions that ( $a$ ) dilute alkali specifically cleaves a glycosidic bond between keratan sulphate and protein without additional cleavage of the polysaccharide chains, and $(b)$ reduction of these alkali-cleaved keratan sulphate chains with $\mathrm{NaB}^{3} \mathrm{H}_{4}$ introduces one ${ }^{3} \mathrm{H}$ atom/polysaccharide chain (Hopwood \& Robinson, 1973, 1974). The ratio of $\bar{M}_{w}$ and $\bar{M}_{n}$ values may be used as an index of polysaccharide polydispersity (Flory, 1953; Hopwood \& Robinson, 1973). Ratios between 1.08 and 1.15 calculated from the molecular-weight values obtained by fractionation of keratan sulphate on Sephadex G-200 (Table 1) indicate that each preparation has a low degree of polydispersity (Hopwood \& Robinson, 1973). The molecular weight of keratan sulphate is found to increase with animal age and to vary considerably from one tissue to another. The molecular weight of keratan sulphate from bovine nasal septum changes from 4000 in the foetus to 7500 in the adult. Similarly the molecular weight of keratan sulphate from bovine intervertebral disc changes from 10000 to 20000 during the equivalent period of development. The degree of sulphation is also found to increase with age (Table 1).

\section{Sugar composition of keratan sulphate preparations}

The composition of each preparation of keratan sulphate was also determined by g.l.c. The results in Table 2 are expressed as mol of monosaccharide/ mol of mannose. Each preparation of KS-A contains about $2 \mathrm{~mol}$ of $\mathrm{N}$-acetylneuraminic acid, whereas preparations KS-P contain about $3 \mathrm{~mol}$ of $N$-acetylneuraminic acid $/ \mathrm{mol}$ of mannose. This difference is consistent with the cleavage of a disaccharide, $N$ acetylneuraminylgalactose, from the keratan sulphate by alkali treatment (Hopwood \& Robinson, 1974).

The sialic acid in adult bovine intervertebral-disc KS-P was identified as $N$-acetylneuraminic acid by paper chromatography and by g.l.c. All of the sialic acid residues in preparation KS-P from adult bovine intervertebral disc are cleaved with neuraminidase (Table 3).

Preparations KS-A and KS-P were also assayed for non-reducing terminal galactose residues by using galactose oxidase as described by Sempere et al. (1965). This reaction is specific for non-reducing terminal galactose or galactosamine. Before neuraminidase treatment, preparations KS-A and KS-P contained negligible amounts of non-reducing 
Table 2. Composition of keratan sulphate preparations determined by g.l.c.

Results are expressed as $\mathrm{mol} / \mathrm{mol}$ of mannose. All samples were cleaved in methanolic $\mathrm{HCl}$ and analysed by g.l.c. as described by Robinson \& Hopwood (1973). Amino acid content was measured with a Beckman Spinco automatic amino acid analyser (Robinson \& Hopwood, 1973) and the results were calculated in terms of the mannose content by equating the glucosamine content in that assay (corrected for loss during hydrolysis) with the $N$-acetylglucosamine content determined by g.l.c. Molecular weights were calculated by assuming $1 \mathrm{~mol}$ of mannose/mol of keratan sulphate and summing all the sugar and amino acid components with due allowance for sulphate content and $\mathrm{Na}^{+}$counterions. KS-A preparations were isolated by alkaline cleavage and KS-P preparations by exhaustive digestion with papain, Pronase and chondroitin sulphate lyase as outlined in the Experimental section. Preparation KS-P-A was isolated after treatment of the corresponding KS-P preparation with alkaline $\mathrm{NaB}^{3} \mathrm{H}_{4}$ as outlined in the Experimental section. Preparation KS-A-A was isolated after hydrolysis of the corresponding KS-A preparation in acetic acid as outlined in the Experimental section. Preparation KS-D was isolated by proteolytic digestion of adult bovine intervertebral disc with papain and Pronase followed by ion-exchange chromatography on Dowex 1 (X4; Hopwood \& Robinson, 1974). This preparation differs from preparation KS-P in that it contains no detectable chondroitin sulphate fragments and is only recovered in $15 \%$ yield. Adult tissues were obtained from steers (2-5 years) within $1 \mathrm{~h}$ of slaughter and foetal tissues were obtained from embryonic calves just before full term. ND, Not determined; nil, not detected; trace, less than $0.05 \mathrm{~mol} / \mathrm{mol}$ of mannose.

\begin{tabular}{|c|c|c|c|c|c|c|c|}
\hline \multirow[b]{2}{*}{$\begin{array}{r}\text { Tissue source } \\
\text { Type of preparation } \\
\text { Composition (mol) }\end{array}$} & \multicolumn{6}{|c|}{ Bovine intervertebral disc } & \multirow{2}{*}{$\begin{array}{c}7 \\
\text { Bovine nasal } \\
\text { septum } \\
\text { Adult } \\
\text { KS-A }\end{array}$} \\
\hline & $\begin{array}{cc}\ldots & \text { Adult } \\
\ldots & \text { KS-A }\end{array}$ & $\begin{array}{l}\text { Adult } \\
\text { KS-P }\end{array}$ & $\begin{array}{c}\text { Adult } \\
\text { KS-P-A }\end{array}$ & $\begin{array}{c}\text { Adult } \\
\text { KS-A-A }\end{array}$ & $\begin{array}{l}\text { Adult } \\
\text { KS-D }\end{array}$ & $\begin{array}{l}\text { Foetal } \\
\text { KS-A }\end{array}$ & \\
\hline $\mathrm{N}$-Acetylglucosamine & 24.3 & 24.1 & 23.8 & 14.2 & 24.2 & 8.2 & 8.5 \\
\hline$N$-Acetylgalactosamine & trace & 5.8 & trace & nil & 0.9 & trace & trace \\
\hline Galactose & 26.4 & 28.2 & 25.2 & 14.0 & 27.2 & 10.1 & 10.0 \\
\hline Mannose & 1.0 & 1.0 & 1.0 & 1.0 & 1.0 & 1.0 & 1.0 \\
\hline Fucose & 0.9 & 1.0 & 0.9 & nil & 1.0 & 0.2 & 0.5 \\
\hline $\mathrm{N}$-Acetylneuraminic acid & 2.2 & 3.0 & 2.1 & nil & 3.1 & 2.1 & 2.1 \\
\hline Glucuronic acid & nil & 4.4 & nil & nil & nil & nil & nil \\
\hline Xylose & nil & 1.0 & nil & nil & nil & nil & nil \\
\hline Amino acid & 13.1 & 13.5 & 6.1 & 7.6 & 9.5 & ND & 15.1 \\
\hline Mol.wt. (calculated) & 14700 & 17700 & - & - & - & - & 6700 \\
\hline
\end{tabular}

Table 3. Enzymic detection of non-reducing terminal monosaccharides in keratan sulphate

Results are expressed as $\mathrm{mol} / \mathrm{mol}$ of keratan sulphate. Values of 18000 and 7500 were used for molecular weights of keratan sulphate from adult bovine intervertebral disc and adult bovine nasal septum respectively. Terminal sialic acid residues were cleaved with neuraminidase and the liberated $N$-acetylneuraminic acid was separated by gel filtration on Sephadex G-50 and assayed chemically by the resorcinol method of Svennerholm (1957). Terminal galactose residues were assayed with galactose oxidase and terminal $\beta$-linked galactose residues were assayed with $\beta$-galactosidase and galactose dehydrogenase as outlined in the Experimental section. Terminal galactose was assayed before and after exhaustive digestion with neuraminidase. Keratan sulphate samples were isolated as described in Table 1. ND, Not determined; nil, not detected; trace, less than $0.05 \mathrm{~mol} / \mathrm{mol}$ of keratan sulphate.

$$
\text { Sample no. } \quad \ldots \quad 1
$$

Tissue source $\quad . . \quad$ Bovine intervertebral disc

Analysis (mol)

Terminal sialic acid

Terminal galactose

Terminal galactose after neuraminidase treatment

Terminal $\beta$-galactoside after neuraminidase treatment $\begin{array}{llll}\text { Type of preparation } \quad . . & \overbrace{\text { Adult KS-A }}^{\text {Adult KS-P }}\end{array}$

2.18

trace

2.06

nil

3.01
trace
2.92
nil

3

Bovine nasal septum Adult KS-A
2.25

trace

2.22

ND terminal galactose, but after neuraminidase treatment these preparations contained approx. 2 and $3 \mathrm{~mol}$ of non-reducing terminal galactose (or galactosamine)/mol of keratan sulphate respectively (Table 3).
Since preparation KS-A contains little or no galactosamine (Table 2), the observed reaction with galactose oxidase indicates that each sialic acid residue in keratan sulphate is linked to a galactose 
residue which has no other substituents. This conclusion is supported by the fact that each KS-A and KS-P preparation shown in Table 2 contains an excess of galactose over $N$-acetylglucosamine equivalent to $2-3 \mathrm{~mol} / \mathrm{mol}$ of keratan sulphate.

The galactose exposed, by neuraminidase treatment of preparations KS-A and KS-P from adult bovine intervertebral disc, is resistant to hydrolytic cleavage by $\beta$-galactosidase from both Escherichia coli and Helix pomatia. It is possible that these galactose residues are linked to keratan sulphate through $\alpha$-glycosidic bonds, or that cleavage with each $\beta$-galactosidase is governed by the surrounding monosaccharide composition and sequence (Hughes \& Jeanloz, 1964; Jungalwala \& Robins, 1968; Seno \& Toda, 1970). Another explanation may be that the $\beta$-galactosidase activity may be considerably decreased by the proximity of charged sulphate groups or by steric hinderance.

One feature common to each keratan sulphate preparation is the presence of mannose (Table 2). The minimum molecular weight of each keratan sulphate preparation, calculated from the analysis shown in Tables 1 and 2 by assuming 1 residue of mannose/polysaccharide chain, closely agrees with the determined molecular weights recorded in Table 1. This result supports the notion that all keratan sulphate preparations contain 1 mannose residue/ chain. Treatment of preparation KS-P from adult bovine intervertebral disc with dilute alkali did not significantly alter the mannose content (Table 2). Hydrolysis of preparation KS-A from bovine intervertebral disc in dilute acetic acid caused con- siderable breakdown of the keratan sulphate structure. When the hydrolysate was subjected to gel filtration on Sephadex G-25 (Fig. 1) significant amounts of radioactivity and hexose were retarded on the column, indicating the release of small oligosaccharide and monosaccharide fragments. The large polysaccharide fragments resulting from this treatment, which were eluted close to the void volume of the column, were isolated by gel filtration on Sephadex G-50 as outlined in the Experimental section and were analysed for radioactivity, amino acid and monosaccharide content (preparation KS-A-A; Tables 2 and 4). The results indicate a complete loss of sialic acid and fucose, an $85 \%$ loss of ${ }^{3} \mathrm{H}$ label but an increase in the amount of mannose associated with the remaining keratan sulphate structure. Unlike the other keratan sulphate preparations assayed, this degraded material contained equimolar amounts of galactose and glucosamine (Table 2).

The ${ }^{3} \mathrm{H}$ label in KS-A preparations acts as a marker for the keratan sulphate reducing end, which is also the region involved in the alkali-labile linkage of keratan sulphate to protein. The substantial increase in mannose content, relative to galactose, together with the considerable decrease of ${ }^{3} \mathrm{H}$ label after hydrolysis in dilute acetic acid (Table 2) suggests that mannose in keratan sulphate is not bonded to the region of the $O$-glycosidic linkage between $\mathrm{N}$-acetylgalactosamine and protein.

The fucose content of keratan sulphate is not constant. Preparations from adult intervertebral disc (KS-A and KS-P), contain about $1.0 \mathrm{~mol}$ of fucose/mol of keratan sulphate, whereas preparations

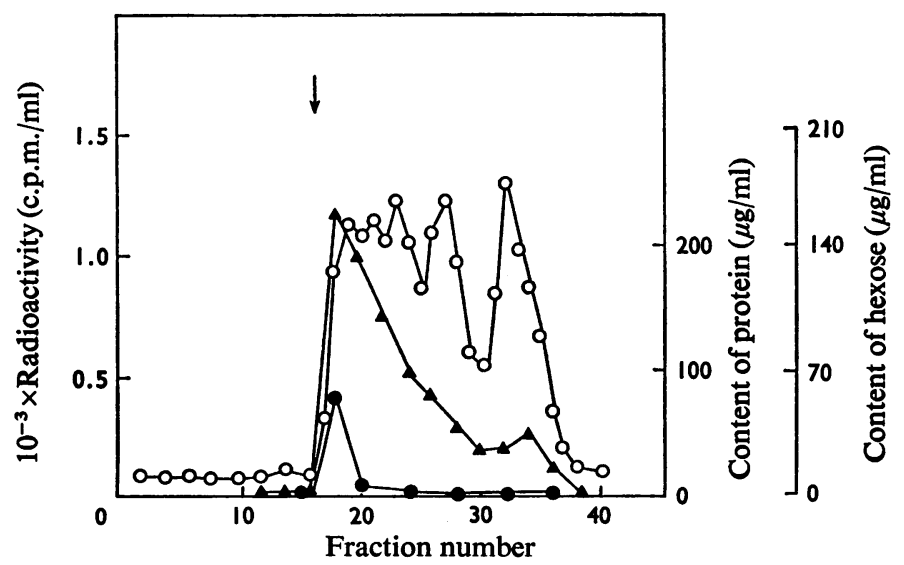

Fig. 1. Gel filtration on Sephadex G-25 of acid-hydrolysed keratan sulphate

Keratan sulphate (preparation KS-A) from bovine intervertebral disc was hydrolysed in $1.0 \mathrm{M}$-acetic acid for $4 \mathrm{~h}$ at $108^{\circ} \mathrm{C}$ under vacuum. The entire hydrolysate was neutralized with pyridine and subjected to gel filtration on Sephadex G-25 as outlined in the Experimental section. Radioactivity $(O)$, hexose content $(\Delta)$ and protein content $(O)$ are shown, The arrow indicates $V_{0}$. 
Table 4. Amino acid composition of keratan sulphate preparations

Results are expressed as mol of amino acid/mol of keratan sulphate. Values of 18000 and 7500 were used for the molecular weights of keratan sulphate from adult bovine intervertebral disc and adult bovine nasal septum respectively. A value of 10000 , which was used for the molecular weight of preparation KS-A-A, was calculated from the mannose content of this preparation. The isolation of preparations KS-A, KS-P, KS-D, KS-P-A and KS-A-A is described in Table 2. Amino acids not shown were present only in trace amounts $(<0.05 \mathrm{~mol} / \mathrm{mol}$ of keratan sulphate).

\begin{tabular}{|c|c|c|c|c|c|c|}
\hline \multirow{2}{*}{\multicolumn{2}{|c|}{$\begin{array}{l}\text { Type of preparation ... KS-A } \\
\text { Amino acid composition (mol) }\end{array}$}} & \multicolumn{4}{|c|}{ Adult bovine intervertebral disc } & \multirow{2}{*}{$\begin{array}{c}6 \\
\text { Adult bovine } \\
\text { nasal septum } \\
\text { KS-A }\end{array}$} \\
\hline & & KS-P & KS-P-A & KS-A-A & KS-D & \\
\hline Asp & 1.55 & 0.72 & 0.53 & 0.46 & 0.65 & 1.77 \\
\hline Thr & 0.88 & 1.70 & 0.56 & 0.55 & 1.52 & 0.91 \\
\hline Ser & 0.78 & 2.52 & 0.88 & 0.81 & 1.03 & 0.86 \\
\hline Glu & 2.10 & 1.82 & 1.37 & 1.47 & 1.58 & 2.42 \\
\hline Pro & 1.55 & 1.61 & 0.78 & 0.81 & 1.52 & 1.76 \\
\hline Gly & 1.44 & 1.70 & 0.63 & 1.32 & 0.98 & 1.68 \\
\hline Ala & 1.05 & 1.13 & 0.49 & 0.73 & 0.57 & 1.20 \\
\hline Val & 0.82 & 0.46 & 0.22 & 0.35 & 0.42 & 0.91 \\
\hline Ile & 0.35 & 0.55 & 0.12 & 0.23 & 0.23 & 0.37 \\
\hline Leu & 0.91 & 0.47 & 0.22 & 0.27 & 0.62 & 1.04 \\
\hline Tyr & 0.22 & 0.10 & 0.06 & 0.17 & 0.08 & 0.24 \\
\hline Phe & 0.56 & 0.53 & 0.19 & 0.25 & 0.32 & 0.66 \\
\hline
\end{tabular}

Table 5. Sialic acid, mannose and fucose contents of subfractions of keratan sulphate from adult bovine intervertebral disc

Keratan sulphate (preparation KS-A) was fractionated by gel-filtration on Sephadex G-200 (Hopwood \& Robinson, 1973). The eluted peak of keratan sulphate was divided into four subfractions and a molecular weight for each subfraction was determined from its specific radioactivity (Hopwood \& Robinson, 1973). Sialic acid, mannose and fucose were measured by g.l.c. as outlined in the Experimental section. Analytical results are expressed as $\%$ by weight of the dry sodium salt of each sample. The values in parentheses indicate the same results expressed as mol of sugar/mol of keratan sulphate.

$\begin{array}{lcccc}\begin{array}{l}\text { Keratan sulphate subfraction } \\ \quad \text { from Sephadex G-200 }\end{array} & 1 & 2 & 3 & 4 \\ \begin{array}{l}\text { Composition (\%) } \\ \text { Sialic acid }\end{array} & & & & \\ \text { Mannose } & 2.32(2.12) & 2.90(2.15) & 4.25(2.20) & 6.00(2.12) \\ \text { Fucose } & 0.65(1.02) & 0.67(0.85) & 1.08(0.96) & 1.72(1.05) \\ 10^{-3} \times \text { Mol.wt. } & 0.53(0.91) & 0.70(0.98) & 0.95(0.93) & 1.49(0.95) \\ & 28.3 & 23.0 & 16.0 & 11.0\end{array}$

from adult bovine nasal septum (KS-A) and from foetal bovine intervertebral disc (KS-A) yield values of 0.5 and $0.2 \mathrm{~mol} / \mathrm{mol}$ respectively. Hydrolysis with dilute acid effectively removed fucose from keratan sulphate (Table 2).

Keratan sulphate (KS-A) from adult bovine intervertebral disc was fractionated by gel filtration on Sephadex G-200 (Hopwood \& Robinson, 1973). The material was retarded by the gel and eluted as a single broad peak which was divided into four subfractions, each of which was assayed for monosaccharide composition. The average molecular weight of each subfraction was estimated from its specific radioactivity by the end-group method of Hopwood \& Robinson $(1973,1974)$. Table 5 shows that the molar content of sialic acid, of mannose and of fucose is relatively constant from one subfraction to another, despite a large change (11000 to $28000)$ in their average molecular weights.

\section{Amino acid composition of keratan sulphate prepara- tions}

Tables 1, 2 and 4 show protein and amino acid content of each keratan sulphate preparation. With keratan sulphate from adult bovine intervertebral disc dilute alkali failed to remove peptide material equivalent to approx. 13 amino acids/keratan sulphate chain. Even after extensive digestion with Pronase and papain and after alkali treatment, approx. 6 amino acid residues remain bonded to each keratan sulphate chain. The content of amino acid is 
not lowered by further digestion with carboxypeptidase A or with leucine aminopeptidase. If this peptide material is covalently linked to keratan sulphate then glutamic acid appears to be the only amino acid present in sufficient amount to be involved in linkage with the polysaccharide. Table 4 shows that serine and threonine are the amino acids destroyed to the greatest extent on alkali digestion of keratan sulphate (KS-P) from adult bovine intervertebral disc.

Amino acid analyses of a number of keratan sulphate preparations are shown in Table 4. Keratan sulphate (KS-P-A) derived by alkali treatment of a KS-P preparation exhibits a different amino acid composition from that found in the untreated KS-P preparation. Five amino acids, threonine, serine, proline, glycine and alanine, undergo a considerable fall in amounts (Table 4). The threonine and serine residues are undoubtedly those involved in the linkage of keratan sulphate and chondroitin sulphate to peptide, whereas the large amounts of proline, glycine and alanine lost after alkali treatment probably reflect the close proximity of these amino acid residues to the linkage serine and threonine residues.

Approx. $15 \%$ of the keratan sulphate chains in preparation KS-P, derived from adult bovine intervertebral disc, was separated from chondroitin sulphate by chromatography on Dowex 1 (Hopwood \& Robinson, 1974). This fraction (KS-D; Table 4) has a significantly lower content of glycine, serine and alanine than does preparation KS-P from the same tissue. Treatment of this keratan sulphate fraction with dilute alkali resulted in the loss of 1.04 threonine residues and only 0.18 serine residue/keratan sulphate chain.

\section{Discussion}

The results presented in this paper are consistent with the suggestion that two different bonds are involved in the linkage of skeletal keratan sulphate to protein. One is an alkali-labile glycosidic bond between $\mathrm{N}$-acetylgalactosamine and threonine; the other appears to be an alkali-stable bond between glutamic acid (or glutamine) and an unknown residue in the keratan sulphate structure. Many studies have indicated the involvement of glutamic acid in an alkali-stable linkage between keratan sulphate and protein (Mathews \& Cifonelli, 1965; Meyer, 1970; Pedrini, 1969; Heinegård, 1972; Hascall \& Riolo, 1972), but the chemical nature of the linkage remains unknown. A possible structure might be an amide bond between a carboxyl group of glutamic acid and the amino group of glucosamine.

The occurrence of two bonds linking keratan sulphate to protein raises further questions about the organization of keratan sulphate chains in inter- vertebral-disc proteoglycan. One possible model might consist of two separate species of keratan sulphate, one linked at the reducing end of the chain by the alkali-labile bond to serine or threonine and another linked at the reducing end of the chain by an alkali-stable bond to glutamic acid. Such a model seems improbable because the molecular weight of keratan sulphate determined by the end-group assay with $\mathrm{NaB}^{3} \mathrm{H}_{4}$ is essentially the same as that obtained by equilibrium sedimentation (Table 1). Since it appears that the end-group assay method is valid only for an alkali-labile linkage (Hopwood \& Robinson, 1973, 1974) and since the molecular-weight distribution of intervertebral-disc keratan sulphate is not markedly polydisperse, the presence of two separate keratan sulphate species would be expected to yield a molecular weight by the end-group assay method approximately twice that determined by equilibrium sedimentation. The close agreement of the two determinations therefore makes this model unsuitable. A structure in which a single species of keratan sulphate is linked to protein at the reducing end of the chain via serine and at another site via glutamic acid is therefore more likely to represent the real structure.

Several investigators have studied the amino acid sequence around the serine residue at the site of chondroitin sulphate linkage. Katsura \& Davidson (1966) found the sequence -Glu-Gly-Ser-Gly- in chondroitin sulphate isolated from pig costal cartilage. Mathews (1971) proposed the partial sequence -Gly-Ser-Gly....Gly-Ser- for chondroitin sulphate doublets isolated from a variety of connective tissues, whereas Johnson \& Baker (1973) have proposed the sequence -Glu-Ala-Ser-Gly-for the site of linkage of chondroitin sulphate in bovine nasal septum.

The significant loss of proline, glycine, alanine, serine and threonine observed after treatment of preparation KS-P with alkali (Table 4) is consistent with the latter sequence and with the overall sequence -Glu-Ala-Ser-Gly-Pro-Thr- now proposed for the keratan sulphate-chondroitin sulphate doublet shown in Fig. 2. Chondroitin sulphate is linked to serine and keratan sulphate to threonine. Fig. 2 shows a second peptide segment linked to keratan sulphate via glutamic acid at a point removed from the reducing end of the chain. This second peptide, which appears to contain serine and proline in close proximity to the linkage glutamic acid (Table 4), remains covalently bonded to the keratan sulphate after alkali treatment. Preparations of keratan sulphate (KS-A) isolated by alkaline cleavage without proteolysis contain amino acids presumably linked via this second alkalistable bond. Preparations of KS-A from bovine intervertebral disc and bovine nasal septum have similar amino acid compositions (Table 4), with high contents of aspartic acid, threonine, serine, 


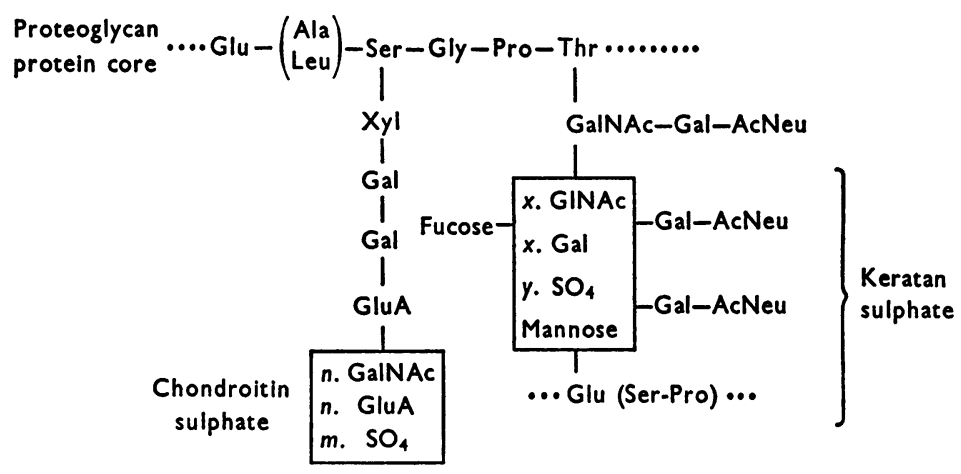

Fig. 2. Proposed structure for chondroitin sulphate-keratan sulphate-protein unit

For adult bovine intervertebral disc the estimated values of $x, y, m$ and $n$ are 24, 31, 25 and 28 respectively. These values were calculated from the sulphate content of each glycosaminoglycan (Table 1; J. J. Hopwood \& H. C. Robinson, unpublished work) and by using molecular weights of 14700 for keratan sulphate (Table 2) and 13500 for chondroitin sulphate chains (Hopwood \& Robinson, 1973). Abbreviation: AcNeu, $N$-acetylneuramic acid.

glutamic acid, proline, glycine, alanine, valine and leucine together with smaller quantities of isoleucine, tyrosine and phenylalanine. No doubt the alkali treatment causes some random breakdown of the polypeptide chain around the alkali-stable linkage region, but the analysis results indicate a fairly common fragment containing an average of 12-14 amino acid residues. The results provide no evidence to suggest that the peptide fragments shown in Fig. 2 are part of a common proteoglycan protein core.

Snellman (1963) isolated a glycopeptide fraction from spleen reticulin, which contained glucosamine, galactose and mannose. He proposed that this glycopeptide consists of two separate peptide chains connected by bridges of oligosaccharides and he suggested that this structure, which is strongly bound to collagen, functions in the organization of collagen fibres. It is possible that keratan sulphate may crosslink proteoglycan units to increase its molecular size (Tsiganos \& Muir, 1969) or provide sites for linkage between proteoglycans, glycoproteins and collagen fibres. These cross-links could result from proteinprotein interaction between other connective-tissue components and a polypeptide linked to keratan sulphate via the alkali-stable bond.

The structure proposed in Fig. 2 also summarizes the probable structural relation of the minor sugars present in keratan sulphate. There are on average 3 molecules of $N$-acetylneuraminic acid linked at nonreducing terminal positions in each keratan sulphate chain through separate galactose units. Treatment of keratan sulphate with dilute alkali releases 1 molecule of $N$-acetylneuraminylgalactose/keratan sulphate chain from the alkali-labile linkage region
(Hopwood \& Robinson, 1974). Although keratan sulphate preparations (KS-A) from foetal bovine intervertebral disc and adult bovine nasal septum have a higher percentage concentration of sialic acid than that of adult bovine intervertebral disc, the sialic acid content expressed as $\mathrm{mol} / \mathrm{mol}$ of keratan sulphate is approx. 2 for each preparation. A similar relationship between sialic acid content and keratan sulphate molecular weight was observed with fractions of preparation KS-A from adult bovine intervertebral disc eluted from Sephadex G-200, where fractionation had occurred on a basis of polysaccharide molecular weight.

If there are indeed 3 sialic acid residues/keratan sulphate chain and if the sialic acid content of highly purified proteoglycan preparations is only that associated with keratan sulphate, it is possible to calculate an approximate molecular weight for keratan sulphate in each preparation from its sialic acid content. An obvious danger with this calculation is that the labile sialic acid residues may be lost during isolation of the keratan sulphate. Luscombe \& Phelps (1967a) reported the sialic acid content of adult bovine nasal septum proteoglycan, which is consistent with keratan sulphate of mol.wt. 8000. The sialic acid content of keratan sulphate from adult bovine nasal septum reported by Gregory \& Rodén (1961) indicates mol.wt. 8000 . Values of 6500 and 7500 respectively are obtained from the results of Hascall \& Riolo (1972) and Hascall et al. (1972) for bovine nasalseptum proteoglycan. The molecular weights of keratan sulphate from bovine nucleus pulposus (Rosenberg et al., 1967) and for keratan sulphate from human intervertebral disc (Podrazky et al., 1970) 
were calculated to be 12000 and 18000 respectively. These calculated values reasonably agree with the molecular weights of 7500 and 18000 recorded in Table 1 for keratan sulphate isolated from adult bovine nasal septum and intervertebral disc.

The concentration of mannose, another minor constituent of all cartilage keratan sulphate, also varied in such a manner that there was approx. 1 residue/keratan sulphate chain. Meyer (1970) and Baker et al. (1969) have suggested that mannose is positioned near the alkali-labile bond between $N$ acetylgalactosamine and threonine or serine in keratan sulphate from human rib cartilage. The results given in the present paper for adult intervertebral-disc keratan sulphate suggest that mannose is located at a position separate from the alkalilabile linkage region, since hydrolysis of KS-A preparations with acetic acid results in no loss of mannose but a considerable loss of ${ }^{3} \mathrm{H}$ label associated with the $\mathrm{N}$-acetylgalactosamine residue involved in linkage to threonine.

The number of fucose residues/keratan sulphate chain, unlike those of the mannose and sialic acid residues, showed considerable dependence on the tissue source and the age of the animal. Perhaps the most noteworthy difference is that between foetal and adult preparations, since there is five times as much fucose/mol of keratan sulphate in the adult intervertebral disc. The addition of fucose and sialic acid residues might function as terminating signals for the carbohydrate chain or trigger the release of proteoglycan from the cell, as has been suggested for glycoproteins in general (Eylar, 1965; Gottschalk, 1969; Carlson et al., 1970).

It is noteworthy that keratan sulphate chains from cartilage each have 3 sialic acid residues and up to 1 fucose residue occupying non-reducing ends. This indicates that keratan sulphate, particularly lowmolecular-weight preparations (e.g. from nasal septum), must have a branched structure. This observation is consistent with the finding that keratan sulphate, when compared with chondroitin sulphate of similar molecular weight, is eluted at a more retarded position from a column of Sephadex G-200 (Hopwood \& Robinson, 1973).

The presence of fucose, mannose, sialic acid, the $\mathrm{O}$-glycosidic linkage to protein through $\mathrm{N}$-acetylgalactamine and the branched nature of keratan sulphate are typical of glycopeptides. Sulphated glycoproteins that appear to be chemically related to cartilage keratan sulphate have been isolated from a variety of tissue sources (Inoue \& Yosizawa, 1966; Pamer et al., 1968; Bignardi et al., 1964; Meyer et al., 1967; How \& Higginbotham, 1970a,b,c). The peptide-carbohydrate linkage region of both cartilage keratan sulphate and the sulphated glycoprotein from chick allantoic membrane when degraded by dilute alkali yield similar products
(Bray et al., 1967; Hopwood \& Robinson, 1974; How \& Higginbotham, 1970c). These sulphated allantoic glycopeptides are antigenic which suggests that keratan sulphate with its similar chemical structure may also function as a connective tissue antigen.

This work was supported by the Australian Research Grants Committee (Project no. D65/15893). J. J. H. thanks Monash University for a Monash Postgraduate Research Scholarship.

\section{References}

Baker, J. R., Cifonelli, J. A., Mathews, M. B. \& Rodén, L. (1969) Fed. Proc. Fed. Amer. Soc. Exp. Biol. 28, 605

Bhavanandan, V. P. \& Meyer, K. (1966) Science 151, 1404-1405

Bhavanandan, V. P. \& Meyer, K. (1967) J. Biol. Chem. 242, 4352-4359

Bhavanandan, V. P. \& Meyer, K. (1968) J. Biol. Chem. 243, 1052-1059

Bignardi, C., Aureli, G., Balduini, C. \& Castellani, A. A. (1964) Biochem. Biophys. Res. Commun. 17, 310-312

Bray, B. A., Lieberman, R. \& Meyer, K. (1967) J. Biol. Chem. 242, 3373-3380

Carlson, D. M., Iyer, R. N. \& Mayo, J. (1970) in Blood and Tissue Antigens (Aminoff, D., ed.), pp. 229-247, Academic Press, London

Eylar, E. H. (1965) J. Theor. Biol. 10, 89-113

Flory, P. J. (1953) Principles of Polymer Chemistry, p. 317, Cornell University Press, New York

Gottschalk, A. (1969) Nature (London) 222, 452-454

Gregory, J. D. \& Rodén, L. (1961) Biochem. Biophys. Res. Commun. 5, 430-434

Hascall, V. C. \& Riolo, R. L. (1972) J. Biol. Chem. 247, 4529-4538

Hascall, V. C., Riolo, R. L., Hayward, J. \& Reynolds, C. C. (1972) J. Biol. Chem. 247, 4521-4528

Heinegård, D. (1972) Biochim. Biophys. Acta 285, 193-207

Hopwood, J. J. \& Robinson, H. C. (1973) Biochem. J. 135, 631-637

Hopwood, J. J. \& Robinson, H. C. (1974) Biochem. J. 141, 57-69

How, M. J. \& Higginbotham, J. D. (1970a) Carbohyd. Res. 12, 355-359

How, M. J. \& Higginbotham, J. D. (1970b) Carbohyd. Res. 14, 327-333

How, M. J. \& Higginbotham, J. D. (1970c) Carbohyd. Res. 14, 335-340

Hughes, R. C. \& Jeanloz, R. W. (1964) Biochemistry 3, 1535-1543

Inoue, S. \& Yosizawa, Z. (1966) Arch. Biochem. Biophys. $117,257-265$

Johnson, H. H. \& Baker, J. R. (1973) Biochem. Soc. Trans. 1, 277-279

Jungalwala, F. B. \& Robins, E. (1968) J. Biol. Chem. 243, 4258-4266

Katsura, N. \& Davidson, E. A. (1966) Biochim. Biophys. Acta 121, 120-127

Lindahl, U. \& Rodén, L. (1965) J. Biol. Chem. 240, 2821-2826 
Lowry, O. H., Rosebrough, N. J., Farr, A. L. \& Randall, R. J. (1951) J. Biol. Chem. 193, 265-275

Luscombe, M. \& Phelps, C. F. (1967a) Biochem. J. 102, 110-119

Luscombe, M. \& Phelps, C. F. (1967b) Biochem. J. 103, 103-109

Mathews, M. B. (1971) Biochem. J. 125, 37-46

Mathews, M.B. \& Cifonelli, J.A.(1965) J. Biol. Chem.240, 4140-4145

Meyer, K. (1969) Amer. J. Med. 47, 664-672

Meyer, K. (1970) in Chemistry and Molecular Biology of the Intercellular Matrix (Balazs, E. A., ed.), vol. 1, pp. 5-24, Academic Press, London

Meyer, K., Bhavanandan, V. P., Yung, D., Lee, L. T. \& Howe, C. (1967) Proc. Nat. Acad. Sci. U.S. 58, 1655-1659

Nakagawa, H. (1971) Nippon Suisan Gakkaishi 37, 921-928

Neurath, H. (1955) Methods Enzymol. 2, 77-83

Pamer, T., Glass, G. B. J. \& Horowitz, M. I. (1968) Biochemistry 7, 3821-3829
Pedrini, V. (1969) J. Biol. Chem. 244, 1540-1546

Podrazky, V., Steven, F. S., Grant, M. E. \& Jackson, D. S. (1970) Biochim. Biophys. Acta 221, 549-558

Robinson, H. C. \& Hopwood, J. J. (1973) Biochem. J. 133, 457-470

Rosenberg, L., Schubert, M. \& Sandson, J. (1967) J. Biol. Chem. 242, 4691-4701

Sempere, J. M., Gancedo, C. \& Asensio, C. (1965) Anal. Biochem. 12, 509-515

Seno, N. \& Toda, N. (1970) Biochim. Biophys. Acta 215, $544-546$

Seno, N., Meyer, K., Anderson, B. \& Hoffman, P. (1965) J. Biol. Chem. 240, 1005-1010

Smith, E. L. (1955) Methods Enzymol. 2, 83-93

Snellman, O. (1963) Acta Chem. Scand. 17, 1049-1056

Svennerholm, L. (1957) Biochim. Biophys. Acta 24, 604-611

Toda, N. \& Seno, N. (1970) Biochim. Biophys. Acta 208, 227-235

Tsiganos, C. P. \& Muir, H. (1969) Biochem. J. 113, 885-894 